\title{
Molecular profiling of transcription factors pinpoints MYC-estrogen related receptor $\alpha$-regulatory factor X5 panel for characterizing the immune microenvironment and predicting the efficacy of immune checkpoint inhibitors in renal cell carcinoma
}

\author{
FEI LIU ${ }^{1 *}$, HONGXIA ZHANG ${ }^{2 *}$, LIHUA XUE $^{3 *}$, QIANKUN YANG ${ }^{4 *}$ and WANCHUN YAN ${ }^{5}$ \\ ${ }^{1}$ Department of Nephrology, The 940th Hospital of Joint Logistics Support Force of Chinese People's Liberation Army; \\ ${ }^{2}$ Department of Emergency Medicine, The First Hospital of The Chinese People's Liberation Army; \\ ${ }^{3}$ Department of Obstetrics and Gynecology, The Family Planning Service Center for Maternal and \\ Child Health in Zhouqu County, Lanzhou, Gansu 730000; ${ }^{4}$ Department of Bone and Soft Tissue, \\ Cancer Hospital of China Medical University, Liaoning Cancer Hospital \& Institute, Shenyang, Liaoning 110042; \\ ${ }^{5}$ Department of Geriatrics, The First Hospital of The Chinese People's Liberation Army, Lanzhou, Gansu 730000, P.R. China
}

Received October 9, 2018; Accepted May 22, 2019

DOI: $10.3892 / \mathrm{ol} .2019 .10523$

\begin{abstract}
Transcription factors (TFs) play key roles in biological processes, and previous studies revealed that they can control oncogenic processes. However, the functional impact of TFs on the prognosis of patients with cancer has not been extensively elucidated. In the context of The Cancer Genome Atlas, few studies have focused on the roles of TFs in tumorigenesis. In the present study, a TF-based robust MYC-estrogen related receptor $\alpha$-regulatory factor X5 (MYC-ESRRA-RFX5) signature was developed for predicting the survival of patients with renal cell carcinoma. Functional enrichment analysis of this signature revealed that it was associated with the immune system of these patients. Further analysis demonstrated that this panel could characterize the immune microenvironment and potentially predicts the effectiveness of immune checkpoint inhibitors. Therefore, the present study recommends future exploration on TF-based biomarkers for their potential as prognostic predictors. Overall, the highlights of this study are: i) This novel study pinpoints a TF panel for the robust prediction of renal cell carcinoma prognosis, and ii) the MYC-ESRRA-RFX5 panel is proposed as a signature for characterizing the immune microenvironment, and to potentially predict the effectiveness of immune checkpoint inhibitors.
\end{abstract}

Correspondence to: Dr Wanchun Yan, Department of Geriatrics, The First Hospital of The Chinese People's Liberation Army, 72 Jingning S Road, Chengguan, Lanzhou, Gansu 730000, P.R. China E-mail: pzsfec5439@126.com

*Contributed equally

Key words: transcription factors, molecular signature, immune, microenvironment, immunotherapy

\section{Introduction}

Transcription factors (TFs), which switch genes on or off, control transcriptional processes (1). By binding to specific genetic sequences, TFs control the molecular hierarchy from DNA to mRNA (2). Alterations in TFs can modify the functions of molecular regulatory networks at multiple levels and hence alter various cell behaviors, such as tumorigenesis and tumor progression (3). For example, the mutation of TF-associated genes can induce dysfunction of TFs and thereby impact the fate of cells (4).

The Cancer Genome Atlas (TCGA; https://portal.gdc. cancer.gov/) has profoundly illuminated the landscape of the molecular networks at different levels and across many cancer types. These analyses, based on high-throughput molecular profiling, provide unparalleled novel insights into cancer (5-7). However, efforts in rewiring the transcriptional regulatory networks are still limited. In particular, it remains unclear how TFs systematically control cancer cells.

Previous fragmentary evidence revealed that dysfunction of TFs cause tumorigenesis and tumor progression (4). For example, signal transducers and activators of transcriptions can activate oncogenic tyrosine kinases and inhibit the functions of oncosuppressors (8). Hence, TFs can serve as therapeutic targets and prognostic molecules in patients with cancer (4). Renal cell carcinoma is the most common type of renal malignancies, accounting for large numbers of new cases and deaths in the United States (9). However, the potential of applying TFs as biomarker panels in renal cell carcinoma has not been extensively studied.

The immune system of a patient partly determines their survival through cancer. The cellular immune system, particularly CD8 $\mathrm{T}$ cells, plays a key role in fighting against cancer. A strong immune system is associated with a better prognosis (10). Immune checkpoint inhibitors, a form of cancer immunotherapy, exert antitumor activity by blocking immune checkpoints [such as cytotoxic T-lymphocyte-associated antigen-4 (CTLA-4), programmed cell death-1 (PD-1) and 
programmed cell death-1 (PD-L1)] and reactivating the immune system (11). Immune checkpoint inhibitors are promising anticancer agents for late stage renal cell carcinoma (11).

There is a clear need for predicting the response of individuals with renal cell carcinoma to immune checkpoint inhibitors. Despite the essential roles of TFs in tumorigenesis, limited efforts have been made to generate prognostic TF panels. Thus, the discovery of potential TF biomarkers is required. Hence, it was compelling to investigate the TF molecular profiles and construct an optimized prognostic model for predicting the survival of patients with renal cell carcinoma.

The present study is the first to demonstrate that prognostic TF panels can robustly predict the survival of patients with cancer, with optimum performance. These findings support the future exploration of TF biomarkers for their potential as prognostic predictors.

\section{Materials and methods}

Data availability. The clinical and RNA sequencing (RNA-seq) data of patients with renal cell carcinoma were downloaded from TCGA (https://cancergenome.nih.gov) and Xena (https://xenabrowser.net/). The differential expression analysis between tumors and normal tissues was not conducted, according to a recent study that reported that cancer vs. normal differential expression analysis did not reveal any prognostic relevance in the context of pan-cancer, and did not identify prognostic genes (12). Data from a total of 537 patients (female, $\mathrm{n}=245$; male, $\mathrm{n}=292$; median age, 61; age range, 26-90) with renal cell carcinoma (Project ID, TCGA-KIRC) were included in the analysis. Ethics approval was not required as data was obtained from an open-access database. The transcription factor regulatory impact was analyzed using RABIT software (version 3; http://rabit.dfci. harvard.edu/download/), the data from which can be downloaded from UCSC Xena (https://xenabrowser.net/datapages/).

Survival analysis and construction of risk score. Multivariate and univariate Cox models, which are widely accepted in the scientific community, were used (13). The cut-off was set at $\mathrm{P}=0.05$. The risk score model formula was constructed based on the Cox coefficient weight: Risk score $=\operatorname{Cox}$ coefficient $_{1} \mathrm{x}$ TF score $_{1}+$ Cox coefficient 2 x TF score $_{2}+\ldots+$ Cox coefficient $_{\mathrm{n}} \times \mathrm{TF}$ score $_{\mathrm{n}}$. A receiver operating characteristics (ROC) curve and the area under the curve (AUC) were generated using R software (version 3.5.2; https://www.r-project.org/). The target genes of the TFs were retrieved from Cistrome Cancer (http://cistrome.org/CistromeCancer/).

Gene set enrichment analysis (GSEA). The enrichment analysis was conducted using mSigDB (http://software.broadinstitute. org/gsea/msigdb/index.jsp) (14). The plots were generated using R software. The Canonical pathway gene sets, Kyoto Encyclopedia of Genes and Genomes (KEGG; https://www.kegg.jp) gene sets and Gene Ontology (GO; http://geneontology.org) gene sets (http://software.broadinstitute.org/gsea/msigdb/genesets.jsp) were used in the present study.

Immune state computation. A precise immunogenomic pipeline was used to characterize the immune states in low- and high-risk groups of patients with renal cell carcinoma (15). The construction of multi-omic gene signatures, based on mRNAs, microRNAs, DNA methylation and copy number, are detailed in a previous study (15). The stromal fraction was computed based on another peer-reviewed publication (16). The cut-off value for high- or low-risk group was based on the median value of the TF score (regulatory impact of each TF calculated by the RABIT tool). Overall survival was used for analysis. Correlation analysis was performed using R software with Spearman's rank test.

Statistical analysis. Comparisons of the transcription factor signatures were performed using unpaired Wilcoxon rank sum test. One-way ANOVA followed by Tukey's post hoc test was used for comparisons between multiple groups. Correlation analysis between PD-L1 expression and risk score was performed using Spearman's correlation test. Cox regression analysis with a cut-off P-value of 0.05 was used for survival analysis screening. $\mathrm{P}<0.05$ was considered to indicate a statistically significant difference.

\section{Results}

Screening TFs. TFs are proven to play key roles in tumorigenesis and tumor progression (17). Clinical and RNA-seq data from TCGA were used in the present study to explore the clinical relevance of TFs in patients with renal cell carcinoma. The regulatory impact of each TF on tumor-specific gene expression patterns was computed using the RABIT tool, now a widely used platform (18). RABIT screened for TFs that impact the gene expression in renal cell carcinoma and selected the most relevant ChIP-seq profile. RABIT further optimized the model, excluding any insignificant TFs. The expression matrix of 90 TFs was calculated and scores of their regulatory impact were obtained.

Cox model confirms the robust signature of TFs. Multivariate and univariate Cox models are currently widely accepted and applied in a number of studies (13). Evidence suggests that Cox models are reliable for identifying prognostic biomarkers in clinical practice $(13,19)$. Hence, the prognostic potential of the 90 TFs was calculated, based on the univariate Cox model analysis. The cut-off was set at $\mathrm{P}=0.05$. As a result, eight $\mathrm{TF}$ biomarker candidates were identified, including T-cell acute lymphocytic leukemia protein 1 , transcription initiation factor TFIID subunit 7, estrogen related receptor $\alpha$ (ESRRA), MYC, regulatory factor X5 (RFX5), Myb-related protein B, sterol regulatory element binding transcription factor 1 and forkhead box A2. Seven of the eight TFs showed different expression patterns between the low- and high-risk groups (Fig. 1).

In order to validate the robustness of the prognostic predictors, their significance as prognostic predictors was determined using the multivariate Cox model and log-rank test. The cut-off value was set at $\mathrm{P}=0.05$, and three TFs (MYC, ESRRA and RFX5) with significant associations with survival were identified (Fig. 2). These three TFs may therefore serve as independent prognosis biomarkers for patients with renal clear cell carcinoma.

The robustness of the three biomarkers as prognostic predictors was tested. The log-rank test was used to 

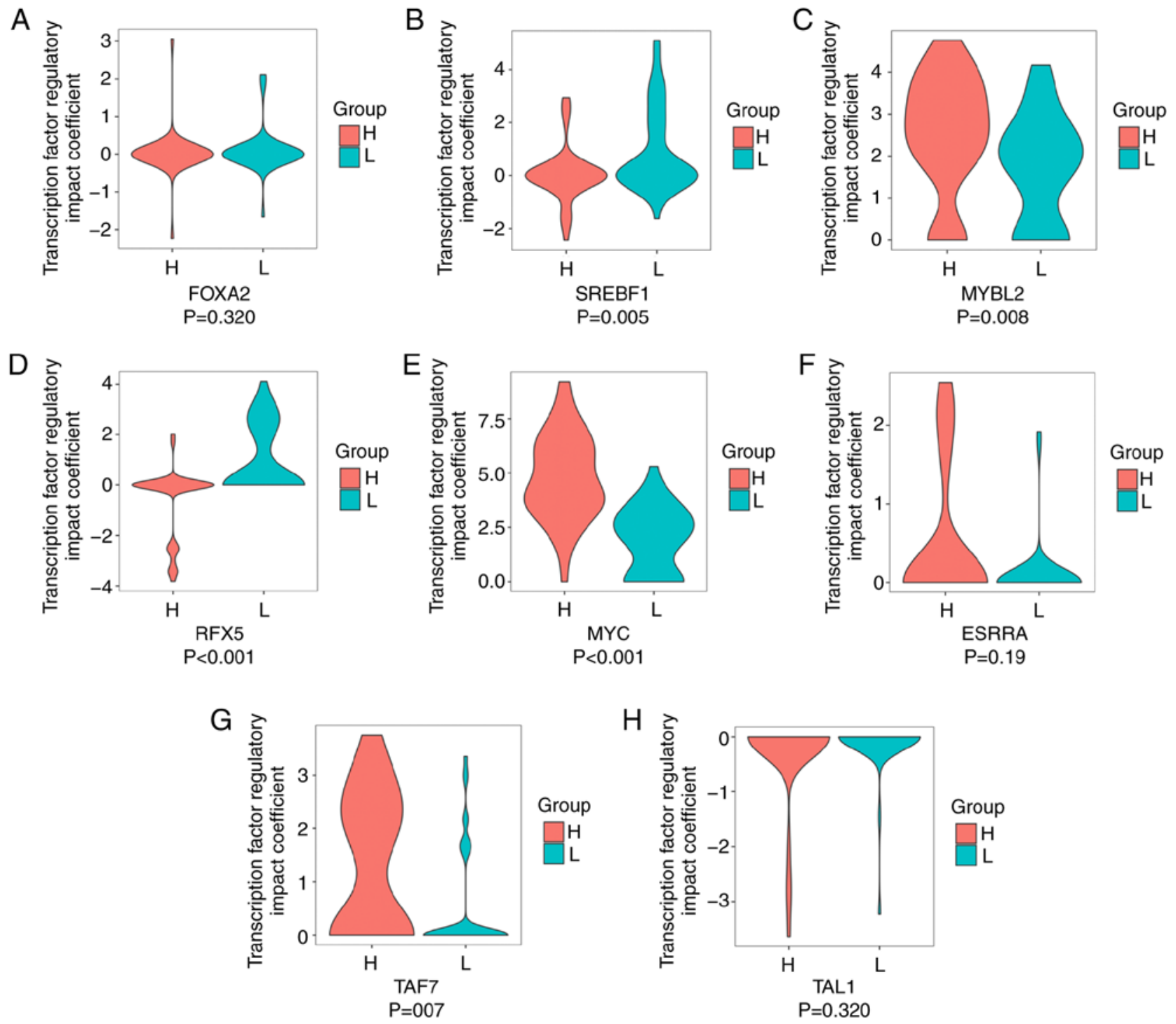

Figure 1. Clinical relevance of the eight candidate TF biomarkers in high- and low-risk groups. Profiles of TF regulatory impact coefficients of (A) FOXA2, (B) SREBF1, (C) MYBL2, (D) RFX5, (E) MYC, (F) ESRRA, (G) TAF7 and (H) TAL1 in L and H groups. P-values were computed through un-paired Wilcoxon rank sum test. TF, transcription factor; L, low-risk; H, high-risk; FOXA2, forkhead box A2; SREBF1, sterol regulatory element binding transcription factor 1; MYBL2, Myb-related protein B; RFX5, regulatory factor X5; ESRRA, estrogen related receptor $\alpha$; TAF7, transcription initiation factor TFIID subunit 7; T-cell acute lymphocytic leukemia protein 1.

demonstrate the differences in the survival between low- and high-risk groups $(\mathrm{P}<0.001$; Fig. $3 \mathrm{~A})$. In order to train the model for optimized robustness, the coefficient weight of each TFs were calculated based on cox regression coefficient. As a result, the MYC-ESRRA-RFX5 signature was constructed (score $=0.75395 \times$ ESRRA+0.24426 x MYC-0.43121 x RFX5). The robustness of the prognostic predictions of this signature was determined using a ROC curve, which generated an AUC value of 0.903 when the overall survival time reached 1 year (Fig. 3B).

Identification of target genes and evaluation of the biological functions of the TF biomarkers. In order to assess the biological significance of this TF signature, gene/pathway enrichment analysis was conducted, to identify and analyze target genes. A similar approach has been applied in investigating the biological roles of miRNAs, which can regulate their target genes $(20,21)$. Thus, a screen for the target genes of the three TFs in the signature was performed. With a focus on renal clear cell carcinoma, the expression of target genes associated with the TFs was analyzed based on an online resource, known as Cistrome Cancer (22). The cut-off for the Spearman's rank correlation test was set at coefficient 0.3 and was applied to determine genes that are positively and negatively correlated with the three TFs. Unexpectedly, no target genes of ESRRA met the established criteria. A few genes with negative correlation were identified, which were excluded from the gene/pathway enrichment analysis due to insufficient data. All genes, excluding ESRRA, were used to conduct the GSEA.

For the pathway enrichment analysis, canonical pathway and KEGG gene sets were used (Fig. 4A; top 10 enriched pathways are listed). Interestingly, the pathway enrichment analysis highlighted several tumorigenesis-associated pathways. The signature-associated pathway included interleukin, which was previously reported to induce tumors in kidney 
A

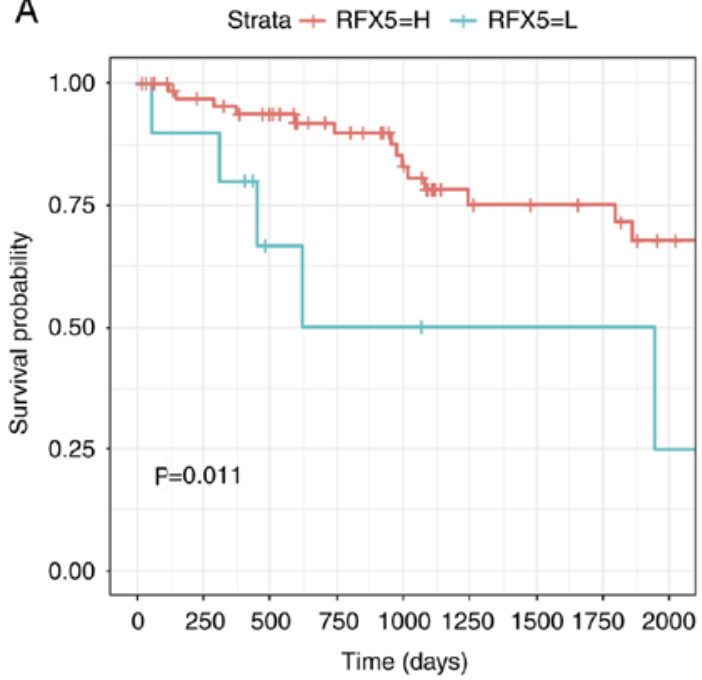

B

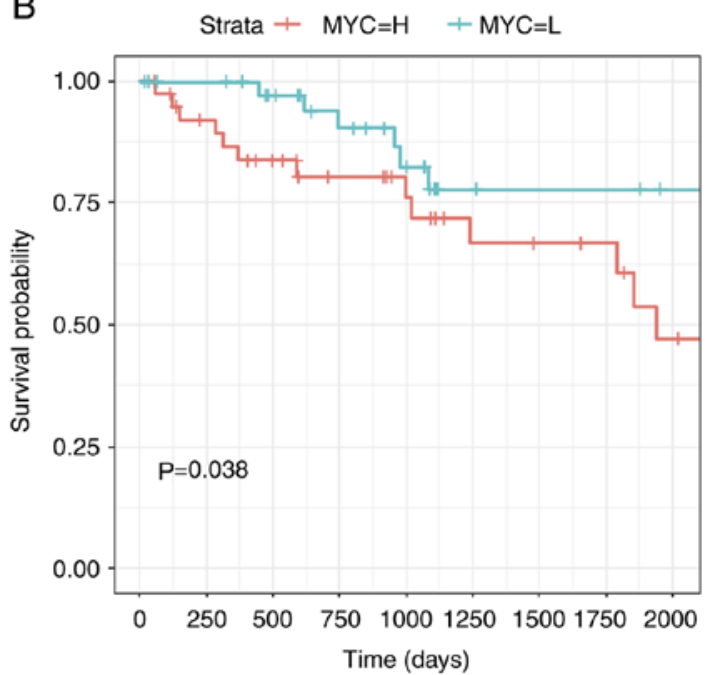

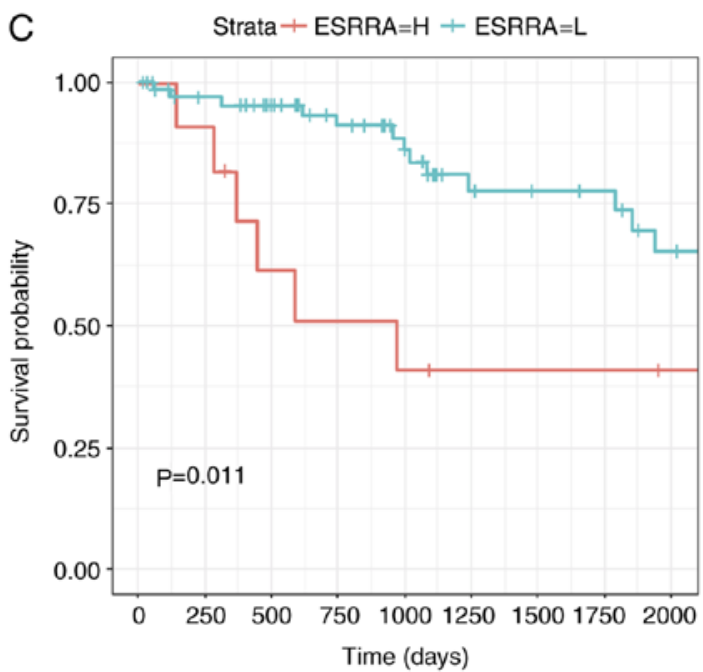

Figure 2. Three transcription factors are associated with the survival of patients with renal cell carcinoma. Survival analysis of patients with $\mathrm{L}$ and $\mathrm{H}$ expression of (A) RFX5, (B) MYC and (C) ESSRA. P-values were computed through the log-rank test. The median cut-off values (regulatory impact of each transcription factor calculated by the RABIT tool) were $0.13,0.93$ and 0.19 for RFX5, MYC and ESSRA survival analysis, respectively. L, low; H, high; RFX5, regulatory factor X5; ESSRA, estrogen related receptor $\alpha$.
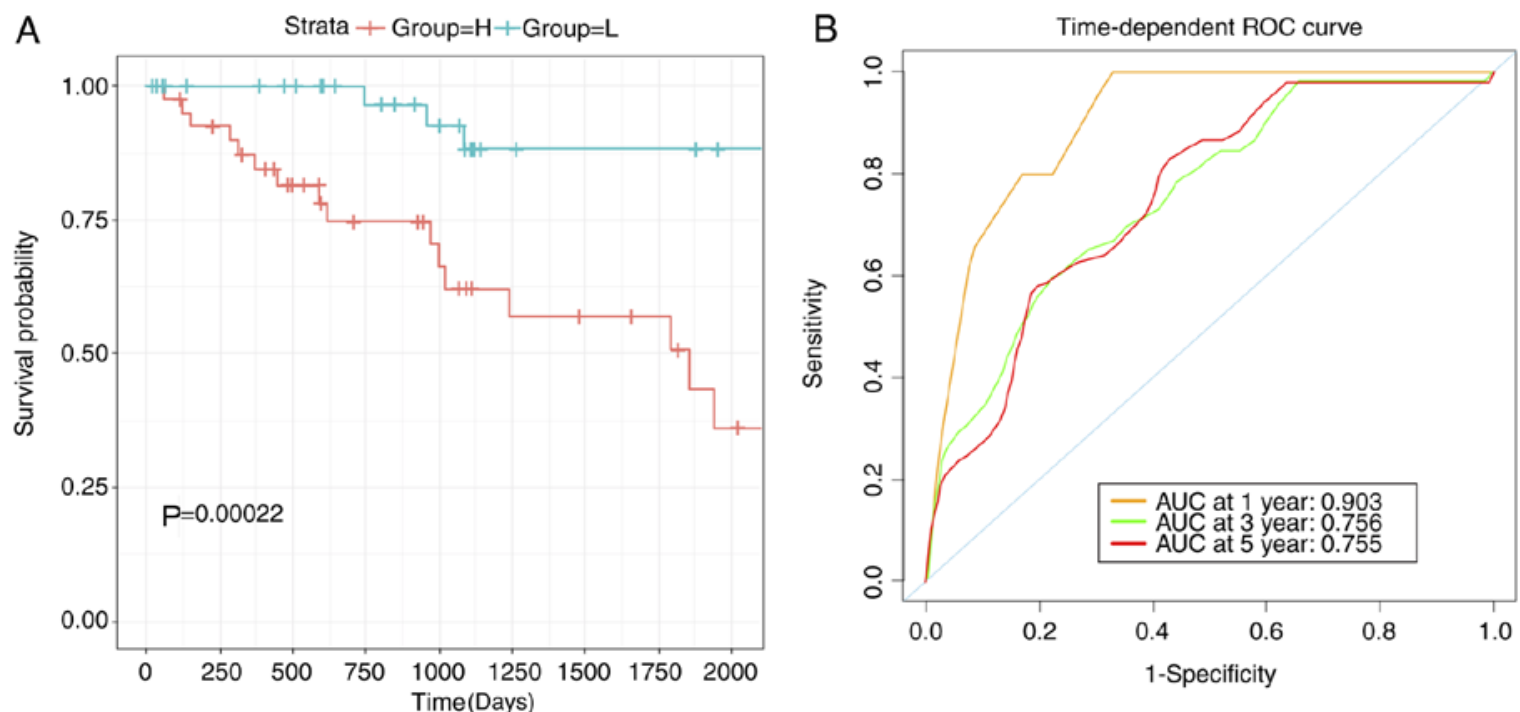

Figure 3. Potential of transcription factor signature as a prognostic predictor. (A) Survival curve of patients with $\mathrm{L}$ and $\mathrm{H}$ expression of the TF signature. (B) ROC curve of the TF signature reveals the robustness of the signature. The median cut-off value for survival analysis was 0.4 (regulatory impact of each TF calculated by the RABIT tool). L, low; H, high; ROC, receiver operating characteristics curve; AUC, area under the curve; TF, transcription factor. 


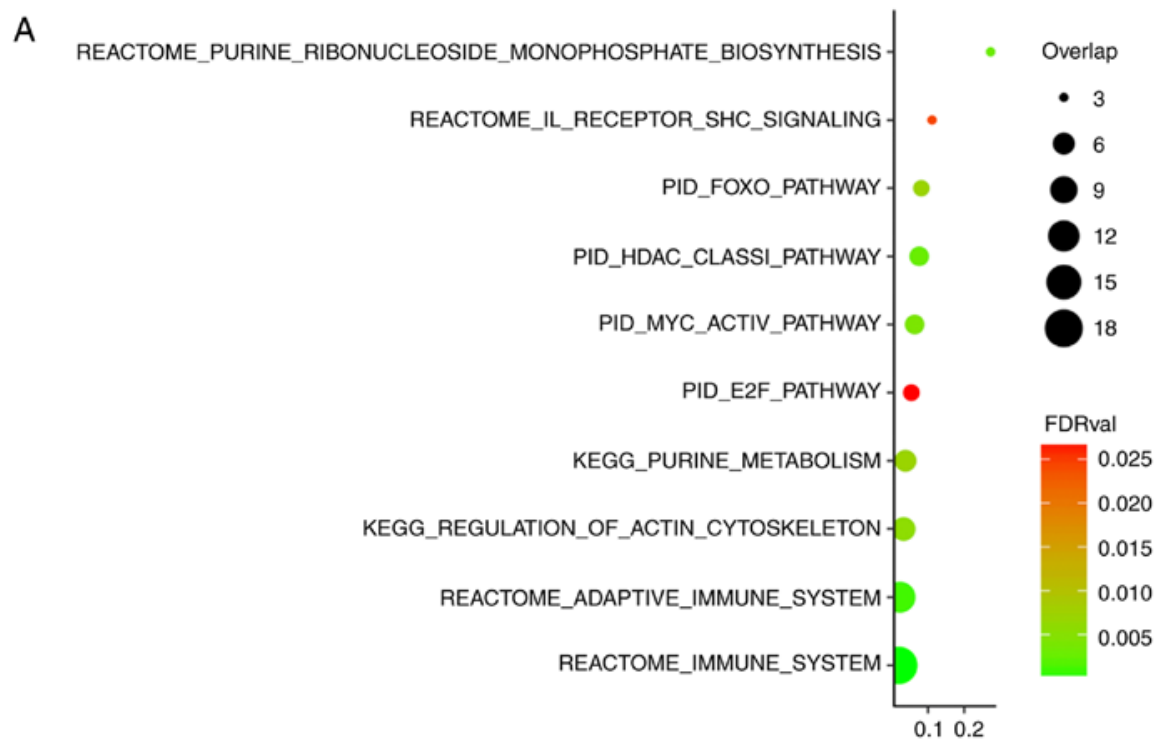

B

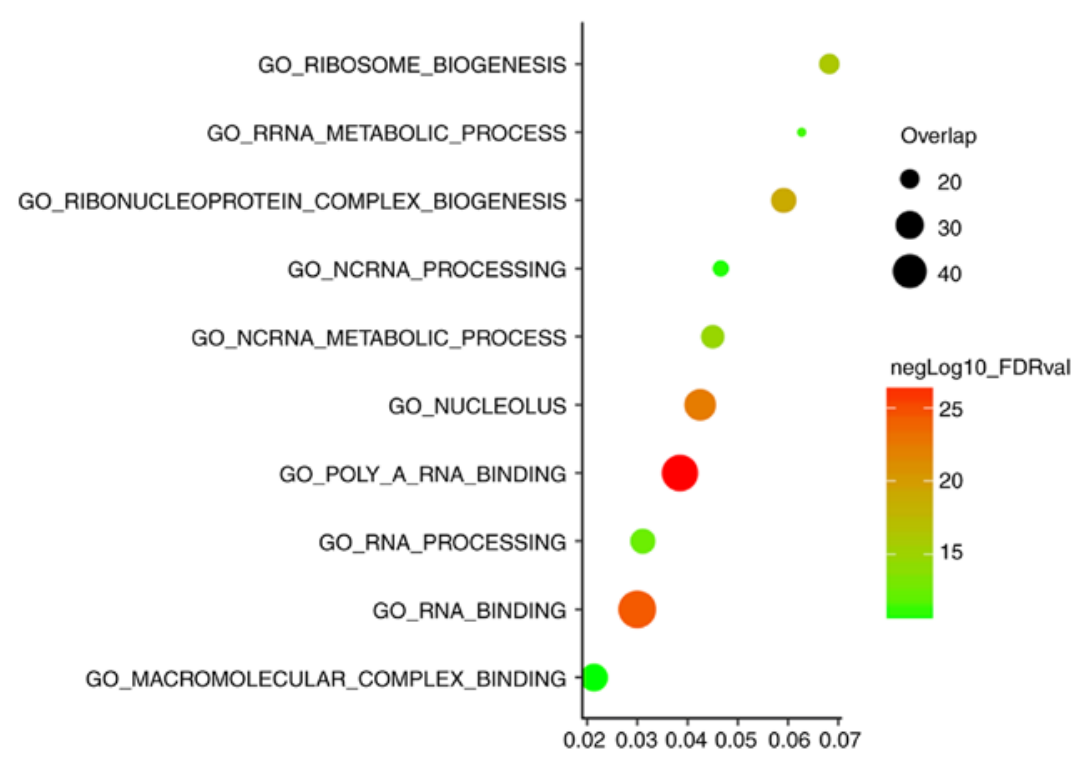

Figure 4. Functional assessment of the transcription factor signature. (A) Pathway enrichment analysis and (B) Gene Ontology enrichment analysis. Overlap represents the enriched genes overlapping with the background gene sets. neglOG10_FDRval represents the negative log10 FDR value of enriched terms.

clear cell carcinoma (23). Alteration of immune system was also highlighted in the pathway enrichment analysis. The GO enrichment analysis was based on the combined analysis of biological processes, cellular components and molecular functions (Fig. 4B, top 10 enriched biological roles are listed). RNA binding was observed as one of the functions, in accordance with the known biological role of TFs (24), demonstrating the credibility of this method of analysis. Another interesting finding was that this TF signature was associated with the metabolic process. This potential biological role requires further experimental validation in association with the MYC-ESRRA-RFX5 signature.

MYC-ESRRA-RFX5 panel can predict the immune cell infiltration level and is associated with the clinical status of the patient. As demonstrated in the biological assessment, the MYC-ESRRA-RFX5 signature may be associated with the immune state of a patient, which is an important indicator of their clinical condition. Based on this finding, the tumor microenvironment of 537 patients was characterized. A study, led by TCGA Research Network, established a reliable immunogenomics pipeline for assessing the immune state of a patient using the immuno-transcriptome (15). This work has paved the way for identifying the precise immune state using data from RNA-Seq. This approach was used to characterize the precise features of the immune state of patients with renal cell carcinoma.

The stromal fraction was first analyzed, as stromal cells are key players in tumor growth, disease development and drug resistance (16). It was found that patients in the low-risk group (classified by the MYC-ESRRA-RFX5 biomarker panel by the median TF value; $n=268$ ) had a median of $\sim 30 \%$ stromal fraction. On the contrary, the high-risk group ( $\mathrm{n}=269)$ had significantly lower stromal fraction (median, $20 \%$; P=0.025; Fig. 5A). Secondly, the lymphocyte infiltration score that marks the general immune state of a patient was determined. The high-risk group displayed significantly lower infiltrated immune cells $(n=269)$ compared with the 

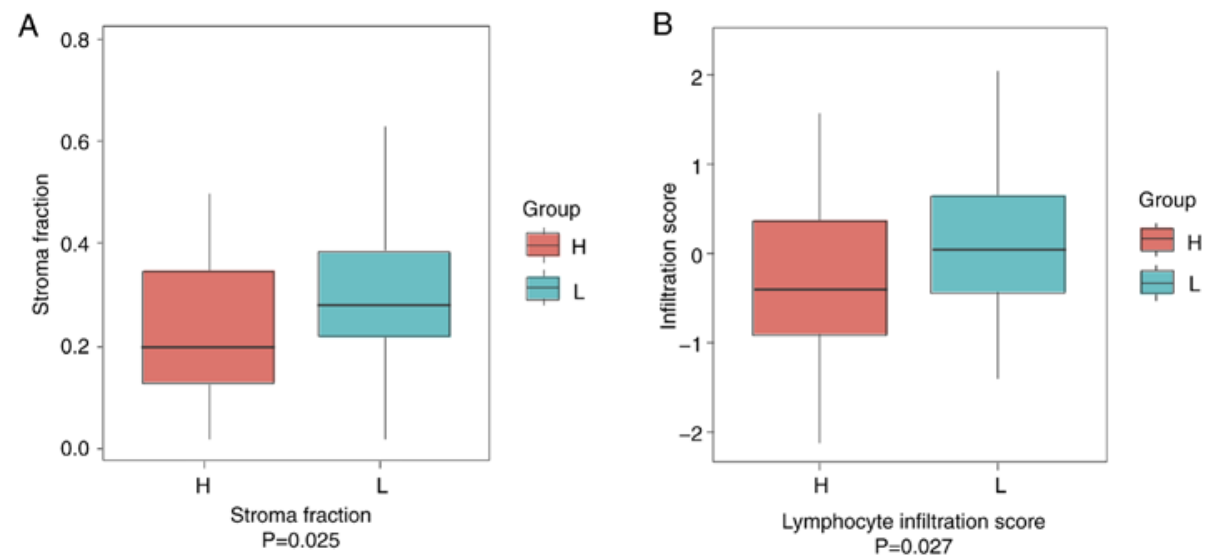

Figure 5. Transcription factor signature can characterize the tumor microenvironment. Comparison of (A) stromal fraction and (B) lymphocyte infiltration score between $\mathrm{L}$ and $\mathrm{H}$ groups. Statistical comparisons were performed using unpaired Wilcoxon rank sum test. L, low-risk; H, high-risk.
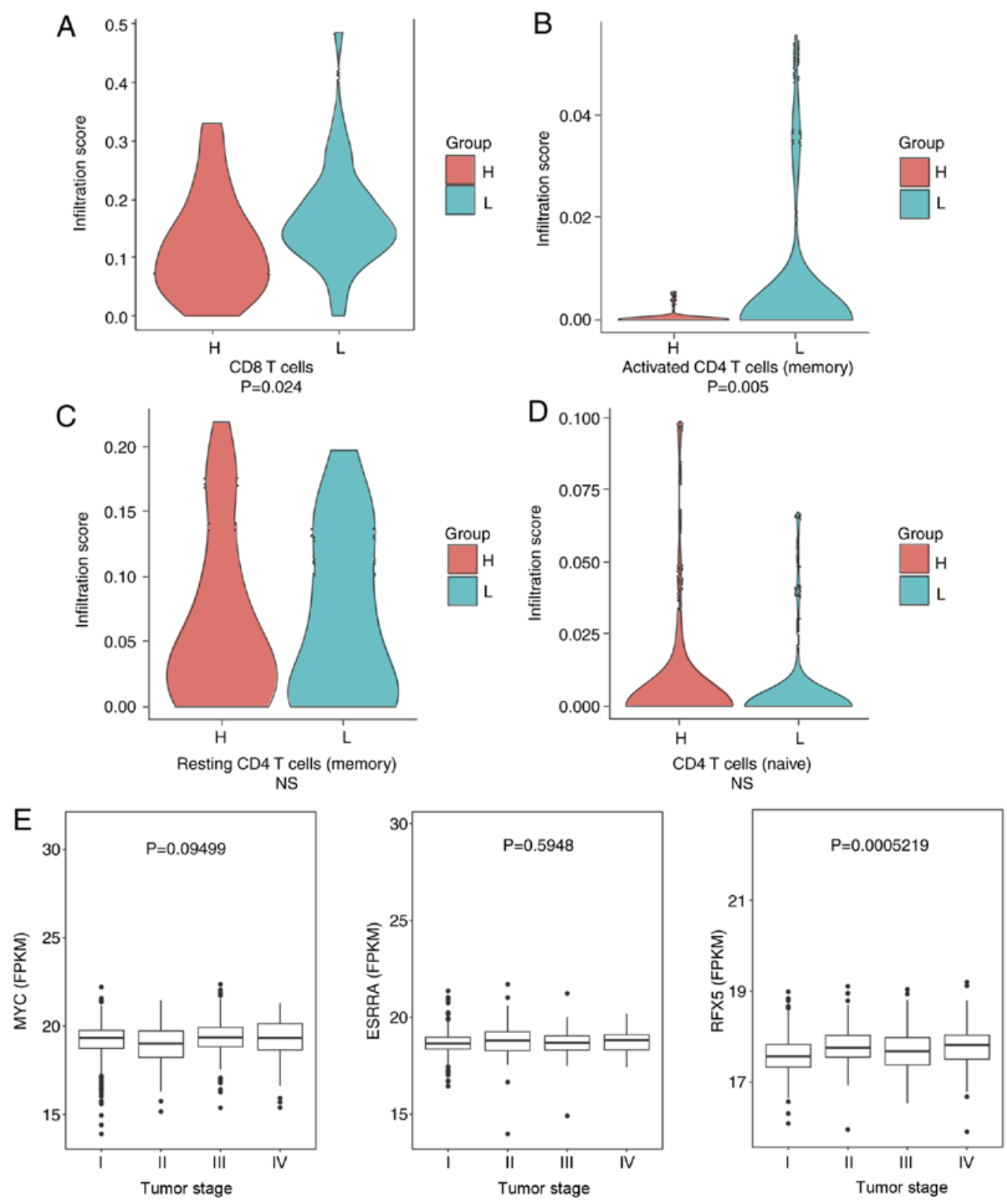

Figure 6. The transcription factor signature can predict the cellular immune state of patients with renal cell carcinoma. Infiltration scores of (A) CD8 T cells, (B) activated CD4 T cells, (C) resting CD4 T cells and (D) naive CD4 T cells in $\mathrm{L}$ and $\mathrm{H}$ groups. P-values were computed through the Wilcoxon rank sum test. (E) Expression of MYC, ESSRA and RFX5 at different clinical stages. P-values were determined using one-way ANOVA followed by a Tukey's post hoc test. L, low-risk; H, high-risk; RFX5, regulatory factor X5; ESSRA, estrogen related receptor $\alpha$; NS, not significant; FPKM, fragments per kilobase million. 
A

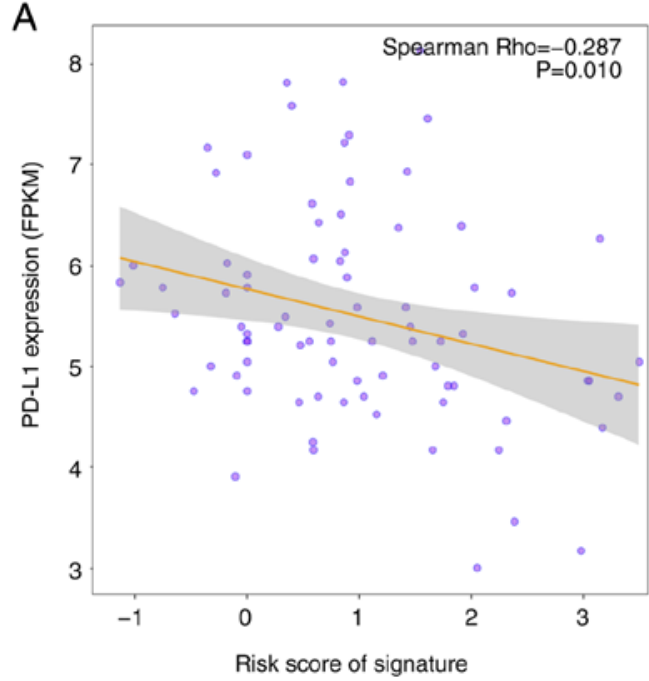

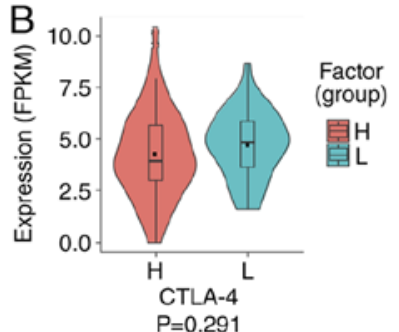
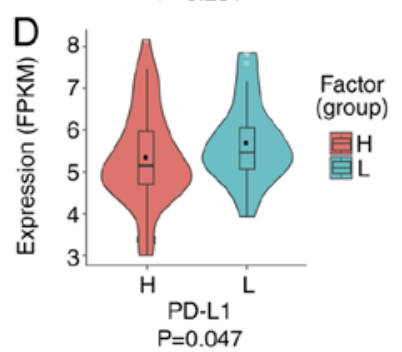
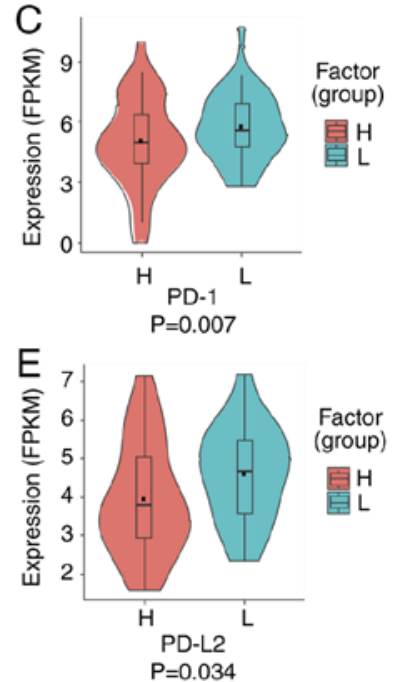

Figure 7. Transcription factor signature may be associated with the effectiveness of immunotherapy. (A) The risk score of the signature was correlated with the PD-L1 expression. The expression of immune checkpoint proteins (B) CTLA-4, (C) PD-1, (D) PD-L1 and (E) PD-L2 were compared between L and $\mathrm{H}$ groups. P-values were computed through the un-paired Wilcoxon signed-rank sum test. The data were compared using Spearman's correlation analysis. L, low-risk; H, high-risk; PD-L1, programmed cell death ligand 1; CTLA-4, cytotoxic T-lymphocyte-associated antigen 4; PD-1, programmed cell death protein 1; PD-L2, programmed cell death ligand 2; FPKM, fragments per kilobase million.

low-risk group ( $\mathrm{n}=268 ; \mathrm{P}=0.027$; Fig. 5B). To evaluate the immune state in more detail, scores of the CD8 $\mathrm{T}$ cells and activated (memory), resting (memory) and naive CD4 T cells were calculated (Fig. 6). Significantly higher infiltration of CD8 T cells and activated CD4 T cells (memory) were observed in the low-risk group $(\mathrm{P}=0.024$ and $\mathrm{P}=0.050$, respectively). The association between the three TFs and the clinical stages was also examined. It was observed that the expression of RFX5 differs between patients at different stages $(\mathrm{P}<0.001$; Fig. 6E). However, MYC and ESRRA were not differentially expressed. These data revealed that RFX5 may potentially serve as a marker of the different stages of renal cell carcinoma.

MYC-ESRRA-RFX5 panel may predict the efficacy of immune checkpoint inhibitors. The expression of PD-L1 is the most commonly used biomarker for predicting response rate and progression-free survival in the clinical use of immune checkpoint inhibitors (PD-1/PD-L1 inhibitors) (25). It was demonstrated that the MYC-ESRRA-RFX5 panel was associated with the immune state of patients with renal cell carcinoma. Thus, the ability of this biomarker panel to predict the effectiveness of PD-1/PD-L1 inhibitors was investigated based on the expression of PD-L1.

First, the correlation between the risk score generated by the MYC-ESRRA-RFX5 panel, and PD-L1 expression was determined. This led to the finding of a significant correlation (Fig. 7A; Spearman's rank coefficient, -0.287; P=0.010). Thereafter, the expression of clinically used immune checkpoint proteins was compared between the low- and high-risk groups. The expression of three of these proteins, PD-1, PD-L1 and PD-L2, was significantly higher in the low-risk group than in the high-risk group (Fig. 7B-E). This may be influenced by the relatively small sample size. This trend suggests that the MYC-ESRRA-RFX5 panel may predict the effectiveness of immune checkpoint inhibitors in clinical practice.

\section{Discussion}

The findings in the present study demonstrated promising prognostic roles of a TF signature that can robustly and efficiently predict survival in renal cell carcinoma. Profiling of TFs was conducted, which led to a three-TF biomarker panel that can predict the survival and immune state of patients with this disease.

Studies on TFs in the context of computational biology are emerging. One such study systematically evaluated the effects of MYC as a transcription factor and demonstrated that MYC interacted with co-regulatory proteins such as MAX, MAX gene-associated protein, MAX dimerization protein, providing new insights into the oncogenic roles of TFs (26). Another study depicted the landscape of the interactions between long non-coding (lnc)RNAs and TFs (8). Notably, GSEA of targets found that lncRNAs indirectly regulate specific TFs. A novel algorithm (named LongHorn) was also developed (8), which precisely predicts the binding sites of lncRNA, TFs and mRNA with high precision. Furthermore, a study by Rau et al (27) explored the gene expression drivers in TGCA tumor samples, and reported on the essential roles of TFs. In the context of cancer treatment, a study recently revealed that targeting the upstream transcriptional factor of PD-L1 is an effective therapy in melanoma (28). Overall, the findings from these studies support the possibility that TFs may serve as targets for cancer therapy.

TCGA is a systematic database that provides data of copy numbers, DNA methylation, RNA-seq, somatic mutations and protein expression based on over 10,000 patients across more than 20 cancer types. Moreover, 27 publications of The Pan-Cancer Genome Atlas unraveled the origin of human cancer, oncogenic processes and tumor-specific signaling pathways from the analysis of over 11,000 tumors across 33 cancer types (29). Among them, two studies focused on TFs in the context of Pan-Cancer (7,26). Interestingly, one study found that 
MYC paralogs were significantly amplified in cancer. It was demonstrated that MYC was associated with immune response signaling, DNA replication and repair function, which was conserved in a number of different types of cancer, including ovarian cancer (26). Another interesting study demonstrated that MYC expression correlates with PD-L1 expression in non-small cell lung cancer, and that tumors expressing both proteins may respond better to immune checkpoint blockade therapy (30). The signature in the present study, containing MYC, also demonstrated an association with immune responses and oncogenic processes. This study highlights the important roles of MYC in renal cell carcinoma.

The present study has some limitations. A major concern is that a validation group is absent, due to insufficient data, despite a thorough search of all available databases of TFs. In addition, a better prognostic model could have been developed using the lasso method (31); however, the AUC value of the lasso method was not better than that based on the Cox model (data not shown). Finally, the correlation between clinical outcome and the MYC-ESRRA-RFX5 panel could not be evaluated due to the lack of patient treatment follow-up data. In the future, further investigation of this TF-based panel in the clinical setting will be conducted.

In summary, the current study suggests an immuneassociated TF panel for predicting the prognosis of patients with renal cell carcinoma. This MYC-ESRRA-RFX5 panel characterizes the immune microenvironment and potentially predicts the effectiveness of immune checkpoint inhibitors.

\section{Acknowledgements}

Not applicable.

\section{Funding}

No funding was received.

\section{Availability of data and materials}

The datasets used and/or analyzed during the current study are available from the corresponding author on reasonable request.

\section{Authors' contributions}

FL, QY, and WY designed the study, analyzed and interpreted the data, and wrote the manuscript. FL, HZ, LX, QY, and WY conducted the data analysis and wrote the manuscript.

\section{Ethics approval and consent to participate}

Not applicable.

\section{Patient consent for publication}

Not applicable.

\section{Competing interests}

The authors declare that they have no competing interests.

\section{References}

1. Iwafuchi-Doi $\mathrm{M}$ and Zaret KS: Pioneer transcription factors in cell reprogramming. Genes Dev 28: 2679-2692, 2014.

2. Filtz TM, Vogel WK and Leid M: Regulation of transcription factor activity by interconnected post-translational modifications. Trends Pharmacol Sci 35: 76-85, 2014.

3. Johnston SJ and Carroll JS: Transcription factors and chromatin proteins as therapeutic targets in cancer. Biochim Biophys Acta 1855: 183-192, 2015.

4. Yeh JE, Toniolo PA and Frank DA: Targeting transcription factors: Promising new strategies for cancer therapy. Curr Opin Oncol 25: 652-658, 2013.

5. Chen H, Li C, Peng X, Zhou Z, Weinstein JN; Cancer Genome Atlas Research Network and Liang H: A pan-cancer analysis of enhancer expression in nearly 9,000 patient samples. Cell 173: 386-399.e12, 2018

6. Sanchez-Vega F, Mina M, Armenia J, Chatila WK, Luna A, La KC, Dimitriadoy S, Liu DL, Kantheti HS, Saghafinia S, et al: Oncogenic signaling pathways in The Cancer Genome Atlas. Cell 173: 321-337.e10, 2018

7. Chiu HS, Somvanshi S, Patel E, Chen TW, Singh VP, Zorman B, Patil SL, Pan Y, Chatterjee SS; Cancer Genome Atlas Research Network, Sood AK, Gunaratne PH and Sumazin P: Pan-cancer analysis of lncRNA regulation supports their targeting of cancer genes in each tumor context. Cell Rep 23: 297-312.e12, 2018.

8. Knaack SA, Siahpirani AF and Roy S: A pan-cancer modular regulatory network analysis to identify common and cancer-specific network components. Cancer Inform 13 (Suppl 5): S69-S84, 2014.

9. Siegel RL, Miller KD and Jemal A: Cancer statistics, 2015. CA Cancer J Clin 65: 5-29, 2015.

10. Binnewies M, Roberts EW, Kersten K, Chan V, Fearon DF, Merad M, Coussens LM, Gabrilovich DI, Ostrand-Rosenberg S, Hedrick CC, et al: Understanding the tumor immune microenvironment (TIME) for effective therapy. Nat Med 24: 541-550, 2018.

11. Massari F, Santoni M, Ciccarese C, Santini D, Alfieri S, Martignoni G, Brunelli M, Piva F, Berardi R, Montironi R, et al: PD-1 blockade therapy in renal cell carcinoma: Current studies and future promises. Cancer Treat Rev 41: 114-121, 2015.

12. An N, Yu Z and Yang X: Expression differentiation is not helpful in identifying prognostic genes based on TCGA datasets. Mol Ther Nucleic Acids 11: 292-299, 2018

13. Tang H, Wang S, Xiao G, Schiller J, Papadimitrakopoulou V, Minna J, Wistuba, II and Xie Y: Comprehensive evaluation of published gene expression prognostic signatures for biomarker-based lung cancer clinical studies. Ann Oncol 28: 733-740, 2017.

14. Subramanian A, Tamayo P, Mootha VK, Mukherjee S, Ebert BL, Gillette MA, Paulovich A, Pomeroy SL, Golub TR, Lander ES and Mesirov JP: Gene set enrichment analysis: A knowledge-based approach for interpreting genome-wide expression profiles. Proc Natl Acad Sci USA 102: 15545-15550, 2005.

15. Thorsson V, Gibbs DL, Brown SD, Wolf D, Bortone DS Ou Yang TH, Porta-Pardo E, Gao GF, Plaisier CL, Eddy JA, et al: The immune landscape of cancer. Immunity 48: 812-830.e14, 2018.

16. Yoshihara K, Shahmoradgoli M, Martínez E, Vegesna R, Kim H, Torres-Garcia W, Treviño V, Shen H, Laird PW, Levine DA, et al: Inferring tumour purity and stromal and immune cell admixture from expression data. Nat Commun 4: 2612, 2013.

17. Liu Z, Dai J and Shen H: Systematic analysis reveals long noncoding RNAs regulating neighboring transcription factors in human cancers. Biochim Biophys Acta 1864: 2785-2792, 2018.

18. Jiang P, Freedman ML, Liu JS and Liu XS: Inference of transcriptional regulation in cancers. Proc Natl Acad Sci USA 112: 7731-7736, 2015.

19. Issa AM, Chaudhari VS and Marchant GE: The value of multigene predictors of clinical outcome in breast cancer: An analysis of the evidence. Expert Rev Mol Diagn 15: 277-286, 2015.

20. Lokeshwar SD, Talukder A, Yates TJ, Hennig MJ, Garcia-Roig M, Lahorewala SS, Mullani NN, Klaassen Z, Kava BR, Manoharan M, et al: Molecular characterization of renal cell carcinoma: A potential three-MicroRNA prognostic signature. Cancer Epidemiol Biomarkers Prev 27: 464-472, 2018.

21. Marziali G, Buccarelli M, Giuliani A, Ilari R, Grande S, Palma A, D'Alessandris QG, Martini M, Biffoni M, Pallini R and Ricci-Vitiani L: A three-microRNA signature identifies two subtypes of glioblastoma patients with different clinical outcomes. Mol Oncol 11: 1115-1129, 2017. 
22. Mei S, Meyer CA, Zheng R, Qin Q, Wu Q, Jiang P, Li B, Shi X, Wang B, Fan J, et al: Cistrome cancer: A web resource for integrative gene regulation modeling in cancer. Cancer Res 77: e19-e22, 2017.

23. Kim HD, Yu SJ, Kim HS, Kim YJ, Choe JM, Park YG, Kim J and Sohn J: Interleukin-4 induces senescence in human renal carcinoma cell lines through STAT6 and p38 MAPK. J Biol Chem 288: 28743-28754, 2013.

24. Cassiday LA: Having it both ways: Transcription factors that bind DNA and RNA. Nucleic Acids Research 30: 4118-4126, 2002.

25. Cyriac G and Gandhi L: Emerging biomarkers for immune checkpoint inhibition in lung cancer. Semin Cancer Biol 52: 269-277, 2018

26. Schaub FX, Dhankani V, Berger AC, Trivedi M, Richardson AB, Shaw R, Zhao W, Zhang X, Ventura A, Liu Y, et al: Pan-cancer alterations of the MYC oncogene and its proximal network across the Cancer Genome Atlas. Cell Syst 6: 282-300.e2, 2018.

27. Rau A, Flister M, Rui H and Auer PL: Exploring drivers of gene expression in the Cancer Genome Atlas. Bioinformatics 35: 62-68, 2019.
28. Zhu B, Tang L, Chen S, Yin C, Peng S, Li X, Liu T, Liu W, Han C, Stawski L, et al: Targeting the upstream transcriptional activator of PD-L1 as an alternative strategy in melanoma therapy. Oncogene 37: 4941-4954, 2018.

29. Blum A, Wang P and Zenklusen JC: SnapShot: TCGA-analyzed tumors. Cell 173: 530, 2018.

30. Kim EY, Kim A, Kim SK and Chang YS: MYC expression correlates with PD-L1 expression in non-small cell lung cancer. Lung Cancer 110: 63-67, 2017.

31. Datta S, Le-Rademacher J and Datta S: Predicting patient survival from microarray data by accelerated failure time modeling using partial least squares and LASSO. Biometrics 63: 259-271, 2007.

(c) (1) (9) This work is licensed under a Creative Commons C. $\mathrm{EY}$ NO Attribution-NonCommercial-NoDerivatives 4.0 International (CC BY-NC-ND 4.0) License. 